\title{
Design and Development of Multiparticulate Floating Drug Delivery System Containing Lisinopril
}

\author{
Rathod Sayali $\mathrm{P}^{1 *}$, Jadhao Umesh $\mathrm{T}^{1}$, Lokhande Sneha $\mathrm{S}^{1}$, Panchal Pranita $\mathrm{P}^{1}$, Wakade $\mathrm{RB}^{2}$
}

${ }^{1}$ Department of Pharmaceutics SDMVM's SVP College of Pharmacy Hatta, Parbhani, M.S Maharashtra, India

${ }^{2}$ Department of Pharmaceutics SNIOP Pusad Dist-Yavatmal M.S Maharashtra, India

DOI: $\underline{10.36347 / \text { sajp.2021.v10i03.004 }}$

| Received: 13.02.2021 | Accepted: 24.02.2021 | Published: 28.03.2021

*Corresponding author: Sayali P. Rathod

Abstract

Original Research Article

The aim of present work is to design multiple unit floating drug delivery system for prolong release of Lisinopril by using different amount of waxes, and to study effect of pectin on buoyancy of the system. The purpose of the study is to prepare wax-encarporated pectin-based emulsion gel beads using a modified Emulsion-gelation technique. The Model drug was incorporated in pectin wax contain olive oil, Lisinopril, were hot-melted, homogenized and then extruded into calcium chloride solution. The prepared Wax-incorporated Emulsion Gel Beads were evaluated for Micromeritics studies, entrapment efficacy, in-vitro buoyancy rate, dissolution rate, it was concluded that the increase of drug release of Lisinopril could be obtained upto $10 \mathrm{hrs}$.Various preformulation studies like bulk density, tapped density, Carr's index angle of repose were in the acceptable limits especially for batch F9. Drug and polymer are compatible with each other as they are verified through FTIR spectroscopy. Increasing the amount of wax in the formulation significantly prolonged the drug release but was insufficient for sustaining the release of highly watersoluble drug.

Keywords: Lisinopril, Multiparticulate, gastroretentive, buoyancy.

Copyright $\odot 2021$ The Author(s): This is an open-access article distributed under the terms of the Creative Commons Attribution 4.0 International License (CC BY-NC 4.0) which permits unrestricted use, distribution, and reproduction in any medium for non-commercial use provided the original author and source are credited.

\section{INTRODUCTION}

Gastro retentive systems or dynamically controlled systems are low-density systems that have comparatively more buoyancy to float over the gastric contents and remain buoyant in the stomach without affecting the gastric emptying time for a prolonged period of rate \& time. This results in an increased gastric retention time and a better control of the fluctuations in plasma drug concentration. Many buoyant systems have been developed based on granules, powders, capsules, tablets, laminated films and hallow Microspheres [1].

Gastro retentive drug delivery system (GRDDS) is one of the gastro retentive dosage forms which could prolong gastric retention time (GRT) to obtain sufficient drug bioavailability. This system floats in the gastric fluid due to its lower bulk density compared to that of the aqueous medium. FDDS is desirable for drugs with an absorption window in the stomach or in the upper small intestine. This system is also useful for drugs which act locally in the proximal part of gastrointestinal (GI) tract, such as antibiotic administration for Helicobacter pylori eradication in the treatment of peptic ulcer andor drugs which are poorly soluble or unstable in the intestinal fluid [2].

Multiparticulate systems show better
reproducible pharmacokinetic behavior than conventional (monolithic) formulations. After disintegration which occurs within a few minutes often even subunits have diameters of less than $2 \mathrm{~mm}$, they are able to leave the stomach continuously, even if the pylorus is closed. These results in lower intra and inter individual variability in plasma levels and bioavailability $[3,4]$.

It would, be advantageous to have means for providing an intimate contact of the drug delivery system with the absorbing membranes. This can be achieved by coupling gastroretentive and bioadhesion characteristics to multiparticulates and developing gastroretentive bioadhesive multiparticulates. These multiparticulates have advantages like efficient absorption and enhanced bioavailability of the drugs due to a high surface to volume ratio, a much more intimate contact with the mucus layer and specific targeting of drugs to the absorption site [5-8]. 
It is stated that, 'the multiparticulates' float on the stomach contents, and then adhere to the mucous linings as the stomach empties. The release of drug from the system can be controlled to coincide with the half-life emptying of the system from the stomach [9$11]$.

\section{MATERIALS AND METHODS Material}

Lisinopril was obtained as kind gift sample from Yarrowchem Pharma, Mumbai India. Pectin purchased from Research lab fine chem. Industries, Mumbai, White wax \& Olive oil was purchased from Thomas baker, Mumbai India. All other materials used of analytical grades.

\section{Methods \\ Preparation of Conventional CaPG Beads and Emulsion Gel Beads}

Conventional CaPG beads were prepared by the ionotropic gelation method $400 \mathrm{mg}$ of pectin were dispersed in water with agitation, and then $100 \mathrm{mg}$ of Lisinopril (80-mesh sieved) were dispersed in pectin solution to make a $100 \mathrm{~g}$ solution. The dispersion was then extruded through a plastic needle into $0.34 \mathrm{M}$ calcium chloride which was gentle stirred at room temperature. The gel beads formed were allowed to stand in the solution for 20 min before being separated and washed with distilled water. The beads were dried at $37^{\circ} \mathrm{C}$ for $12 \mathrm{~h}$.

The emulsion gel beads of calcium pectinate were prepared by emulsion-gelation method $400 \mathrm{mg}$ of pectin were dissolved in water with agitation. $3 \mathrm{ml}$ of olive oil were added to the mixture of pectin and lisinopril (1:1) to make 100-g mixtures and homogenized using a homogenizer, at 3,000 rpm for 5 min. The emulsion gel beads were treated in the same manner as conventional CaPG beads [12-13].

\section{Preparation of Wax-incorporated Emulsion Gel Beads}

Various amounts of different waxes (i.e. white wax and cetyl alcohol) were melted in water bath at 60 $85^{\circ} \mathrm{C}$, depending on the melting ranges of the waxes used. The molten wax was dispersed in the homogenized emulsion mixture of pectin, oil and Lisinopril which already heated to same temperature, and then mixed until the homogenous mixture was obtained. The hot melted mixture was extruded into $0.34 \mathrm{M}$ calcium chloride (cooled at $5^{\circ} \mathrm{C}$ ). The waxincorporated emulsion gel beads obtained were treated in the same manner as conventional CaPG beads. The formulations studied are shown in Table

Table-1: Formulation Table

\begin{tabular}{|l|l|l|l|l|l|l|l|l|l|}
\hline Batches & F1 & F2 & F3 & F4 & F5 & F6 & F7 & F8 & F9 \\
\cline { 1 - 9 } Ingredients (mg) & & & & & & & & & \\
\hline Lisinopril & 100 & 100 & 100 & 100 & 100 & 100 & 100 & 100 & 100 \\
\hline Pectin & 400 & 400 & 400 & 400 & 400 & 400 & 400 & 400 & 400 \\
\hline White wax & 150 & 150 & 150 & 200 & 200 & 200 & 250 & 250 & 250 \\
\hline Cetyl alcohol & 100 & 150 & 200 & 100 & 150 & 200 & 100 & 150 & 200 \\
\hline Olive oil (ml) & 3 & 3 & 3 & 3 & 3 & 3 & 3 & 3 & 3 \\
\hline Distilled water & 20 & 20 & 20 & 20 & 20 & 20 & 20 & 20 & 20 \\
\hline 0.340M Cal. chloride (ml) & 100 & 100 & 100 & 100 & 100 & 100 & 100 & 100 & 100 \\
\hline
\end{tabular}

\section{Evaluation of Lisinopril Beads}

Micromeritics studies of floating beads [14]

The microspheres were characterized by their Micromeritics properties, such as particle size, bulk density, tapped density, Carr's compressibility index, hausner ratio and flow property.
Percentage yield of microspheres formed.

The percentage yield of the prepared microsphere determined by weighing after drying. The measured weight of prepared microspheres was divided by the total amount of all the non-volatile components used for the preparation of the microspheres, which gave the total percentage yield of microspheres.

\section{$\%$ Yield $=$ Actual wt of product $\div$ Total wt of exipient and drug $\times 100$}

\section{Drug Entrapment Efficiency (DEE)}

About $10 \mathrm{~g}$ of beads were weighed accurately and crushed in glass mortar. Powdered beads were suspended in $100 \mathrm{ml}$ of $0.1 \mathrm{~N} \mathrm{HCL}$. After $24 \mathrm{hr}$ the solution was filtered and filtrate was analysed for drug content at $230 \mathrm{~nm}$. The results of \% lisinopril loading and encapsulation efficiency were calculated using Equation. The drug content was calculated from standard curve.

$\%$ Drug entrapment $=$ Calculated drug concentration $\div$ Theorotical drug concentration $\times 100$ 
Rathod Sayali P et al., Sch Acad J Pharm, Mar, 2021; 10(3): 54-59

\section{In vitro buoyancy study: [16-18]}

Appropriate quantity of the floating micro particulate was placed in $100 \mathrm{ml}$ of the simulated gastric fluid (SGF, $\mathrm{pH}$ 2.0), the mixture was stirred with a magnetic stirrer. The layer of buoyant micro particulate was pipetted and separated by filtration. Particles in the sinking particulate layer were separated by filtration. Particles of both types were dried in a desiccator until constant weight was achieved. Both the fractions of microspheres were weighed and buoyancy was determined by the weight ratio of floating particles to the sum of floating and sinking particles.

\section{Buoyancy $(\%)=\mathrm{Wf} / \mathrm{Wf}+\mathrm{Ws} \times \mathbf{1 0 0}$}

Where,

Wf and Ws are the weights of the floating and settled micro particles.

\section{Dissolution test (in-vitro drug release) of microspheres}

The dissolution test was performed using 900 ml $0.1 \mathrm{~N} \mathrm{HCL}$ fluid maintained at $37 \pm 0.50 \mathrm{C}$ and stirred at $100 \mathrm{rpm}$ for $10 \mathrm{hr}$. A sample $(1 \mathrm{ml})$ of the aliquots were withdrawn at different time intervals and an equivalent volume of medium prewarmed at 370C was added to maintain sink condition. The withdrawn samples were filtered through Whatman filter paper no. 41. Absorbance of these solutions was measured at 230 $\mathrm{nm}$. Percentage drug release was calculated using an equation obtained from a standard curve.

\section{Infrared Spectroscopy Interpretation for interaction between drug and polymer in Microsphere}

Fourier transforms infrared spectroscopy (FTIR) spectra of the pure drug and the microspheres were produced using by $\mathrm{KBr}$ disk method. Powder microsphere and the ingredients used in drug loading were subjected to FTIR with a Shimadzu 8201 PC FTIR. Background spectrum was collected before running each sample. The samples were analyzed between wave numbers 4000 and $400 \mathrm{~cm}-1$ [20-22].

\section{Stability Study for Multiple Unit System}

During the stability studies the product is exposed to normal conditions of temperature and humidity. However the studies will take a longer time and hence it would be convenient to carry out the accelerated stability studies where the product is stored under extreme conditions of temperature. In the present study, stability studies were carried out on optimized formulation. The microspheres were stored at $40 \pm 20$ $\mathrm{C} / 75 \pm 5 \% \mathrm{RH}$ for duration of one month. At the interval after thirty days each sample was withdrawn and tested for drug entrapment, and drug release study.

\section{RESULT AND DISCUSSION Micromeritic Properties}

Micromeritic properties for batch F1 to F9 are shown in Table No. 9. The results showed bulk density and Cars index in the range of $12 \%$ to $16 \%$, Hausners ratio less than 1.25 which shows good flow properties. The values were found to be in the range of $21^{\circ} 28^{\prime} \pm 1.4$ to $29^{\circ} 69^{\prime} \pm 1.8$, beads shows the angle of repose less than $30^{\circ}$ which reveals good flow property. The observed results suggest good flow ability of the beads. Bulk density may affect buoyancy of floating beads. The bulk density of formulations was found to be between $0.165 \pm 0.02$ to $0.254 \pm 0.02 \mathrm{~g} / \mathrm{cm}^{3}$. This indicates good packing capacity. The tapped density of beads was found to be in range of $0.197 \pm 0.032$ to $0.308 \pm 0.018 \mathrm{~g} / \mathrm{cm}^{3}$, which shows good packability of beads. The mean particle sizes of all formulations were ranged from $2.04 \pm 0.37 \mathrm{~mm}$ to $2.32 \pm 0.60 \mathrm{~mm}$ as shown in Table No 10. Higher particle size was obtained when the proportion of waxes was increased in polymer and drug mixture of pectin: lisinopril. As the amount of calcium chloride was increased, more crosslinking structure was observed that lead to a decrease in particle size.

Table-2: Micromeritics studies of floating beads

\begin{tabular}{|c|c|c|c|c|c|c|}
\hline $\begin{array}{l}\text { Para-meters } \\
\text { Batches }\end{array}$ & $\begin{array}{r}\text { Bulk } \\
\text { density } \\
\left(\mathrm{g} / \mathrm{cm}^{3}\right)\end{array}$ & $\begin{array}{l}\text { Tapped } \\
\text { density } \\
\left(\mathrm{g} / \mathrm{cm}^{3}\right)\end{array}$ & $\begin{array}{l}\text { Carr's } \\
\text { Index }\end{array}$ & $\begin{array}{l}\text { Hausner } \\
\text { ratio }\end{array}$ & $\begin{array}{l}\text { Angle of } \\
\operatorname{repose}(\theta)\end{array}$ & $\begin{array}{l}\text { Particle } \\
\text { size }(\mathbf{m m})\end{array}$ \\
\hline F1 & $0.246 \pm 0.02$ & $0.308 \pm 0.018$ & $20.12 \pm 0.15$ & $1.25 \pm 0.12$ & $28^{\circ} 65^{\prime} \pm 0.8$ & $2.23 \pm 0.35$ \\
\hline $\mathrm{F} 2$ & $0.165 \pm 0.02$ & $0.197 \pm 0.032$ & $16.24 \pm 0.12$ & $1.19 \pm 0.006$ & $26^{\circ} 50^{\prime} \pm 2.5$ & $2.2 \pm 0.43$ \\
\hline F3 & $0.186 \pm 0.02$ & $0.219 \pm 0.041$ & $15.06 \pm 0.26$ & $1.17 \pm 0.005$ & $29^{\circ} 69^{\prime} \pm 1.8$ & $2.24 \pm 0.35$ \\
\hline F4 & $0.236 \pm 0.03$ & $0.265 \pm 0.027$ & $10.94 \pm 0.18$ & $1.12 \pm 0.006$ & $27^{\circ} 23^{\prime} \pm 1.5$ & $2.22 \pm 0.19$ \\
\hline F5 & $0.222 \pm 0.01$ & $0.255 \pm 0.019$ & $12.94 \pm 0.20$ & $1.14 \pm 0.007$ & $28^{\circ} 65^{\prime} \pm 0.8$ & $2.04 \pm 0.37$ \\
\hline F6 & $0.254 \pm 0.02$ & $0.306 \pm 0.029$ & $16.99 \pm 0.23$ & $1.20 \pm 0.004$ & $26^{\circ} 50^{\prime} \pm 2.1$ & $2.15 \pm 0.222$ \\
\hline F7 & $0.250 \pm 0.03$ & $0.283 \pm 0.04$ & $11.66 \pm 0.21$ & $1.13 \pm 0.002$ & $26^{\circ} 69^{\prime} \pm 2.3$ & $2.32 \pm 0.60$ \\
\hline F8 & $0.217 \pm 0.01$ & $0.261 \pm 0.030$ & $16.85 \pm 0.24$ & $1.20 \pm 0.004$ & $21^{\circ} 28^{\prime} \pm 1.4$ & $2.26 \pm 0.23$ \\
\hline F9 & $0.225 \pm 0.02$ & $0.257 \pm 0.023$ & $12.45 \pm 0.19$ & $1.14 \pm 0.007$ & $27^{\circ} 23^{\prime} \pm 1.2$ & $2.26 \pm 0.20$ \\
\hline
\end{tabular}

\section{Determination of Percentage yield}

It was found that the average percentage yield was greater than $80 \%$ for all formulations. The drug loading was found to be in range of $84.21 \pm 0.5 \%$ to
$97.22 \pm 0.2 \%$. Formulation F1 showed highest loading of $97.22 \pm 0.2 \%$. Whereas formulation F9 showed lowest drug loading of $84.21 \pm 0.5 \%$. Overall the drug loading 
Rathod Sayali P et al., Sch Acad J Pharm, Mar, 2021; 10(3): 54-59

was decreased with increase in the concentration of waxes.

\section{Drug entrapment efficiency}

Ionic gelation technique is convenient method for the preparation of floating beads with good drug loading and encapsulation efficiency. In this method drug is dispersed equally in the polymer matrix so drug can be loaded easily in the polymer. The drug entrapment efficiency of the prepared beads was found to be increased progressively with an increase in concentration of waxes and polymers. This might be due to increased matrix density.

\section{In vitro buoyancy study}

The formulation $\mathrm{F} 1$ gives best floating ability of $92.38 \%$ in $0.1 \mathrm{~N} \mathrm{HCL}$ for $10 \mathrm{hrs}$. This may be due to its low bulk density. The floating ability of the formulations ranges from $65.52 \%$ to $93.20 \%$, which shows the excellent floating ability of the beads. In this as the concentration of wax increases, the viscocity of mixture increases and thus leads to good floating ability.

Table-3: Percentage yield, In vitro buoyancy study, Drug entrapment efficiency

\begin{tabular}{|l|l|l|l|}
\hline Batch & Drug entrapment efficiency $(\boldsymbol{\%})$ & Yield $(\boldsymbol{\%})$ & $(\boldsymbol{\%})$ buoyancy \\
\hline F1 & $86.45 \%$ & $97.22 \pm 0.2$ & $92.38 \%$ \\
\hline F2 & $93.20 \%$ & $94.11 \pm 0.7$ & $84.34 \%$ \\
\hline F3 & $88.67 \%$ & $92.94 \pm 0.2$ & $90.67 \%$ \\
\hline F4 & $76.25 \%$ & $95 \pm 0.4$ & $79.33 \%$ \\
\hline F5 & $65.52 \%$ & $86.87 \pm 0.5$ & $69.32 \%$ \\
\hline F6 & $83.34 \%$ & $91.73 \pm 0.3$ & $78.34 \%$ \\
\hline F7 & $75.38 \%$ & $96.47 \pm 0.3$ & $86.12 \%$ \\
\hline F8 & $76.50 \%$ & $88.88 \pm 0.4$ & $83.78 \%$ \\
\hline F9 & $89.42 \%$ & $84.21 \pm 0.5$ & $77.23 \%$ \\
\hline
\end{tabular}

\section{In vitro drug release study}

It was observed that the beads ascended to the upper part of the dissolution vessels and remains floated until the completion of release studies. The drug release study was carried for $10 \mathrm{hrs}$. This showed as the concentration of waxes increases the release rate of the drug decreases which were attributed to increase density of the polymer matrix at highest concentration causing decreased diffusional path length, and increases drug release from the polymer matrix. Moreover dissolution study data revealed that release from the beads was largely dependent on the polymer swelling and drug diffusion. The percentage drug release from batches F1 to F9 varied from $61.01 \%$ to $96.31 \%$. The in-vitro drug release of the formulation F1 to F9 displayed in Table where comparative release of drug is shown.

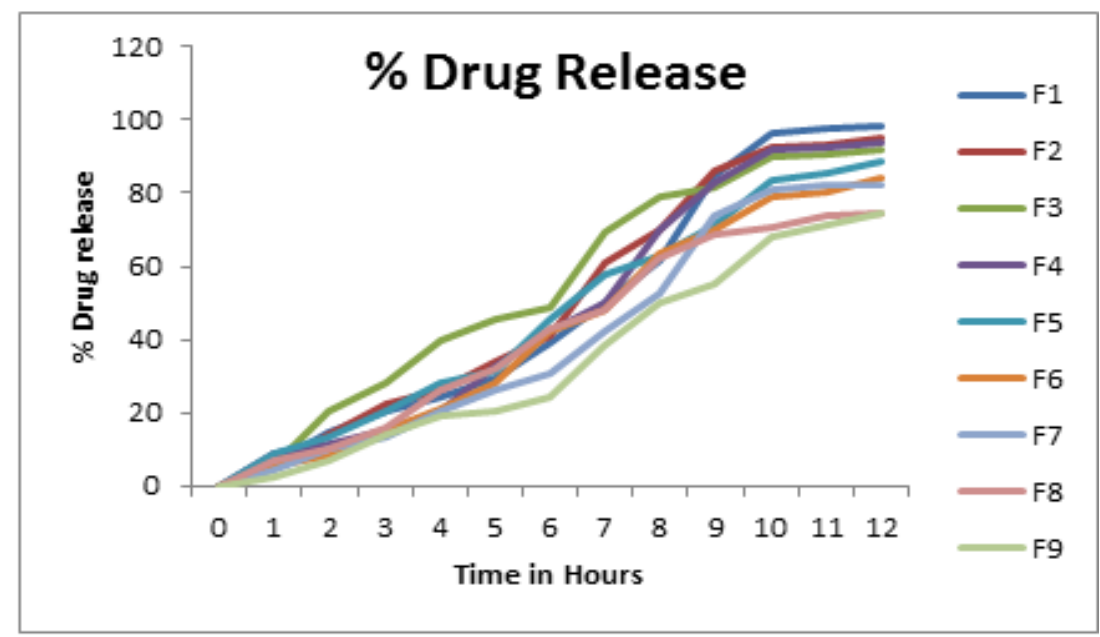

Fig-1: In vitro drug release study

\section{IR Study}

The infrared absorption spectrum of pure lisinopril and the sustained release microsphere of optimized polymer were recorded on FT-IR spectrophotometer (Model-8400S, Shimadzu, Japan) and the spectrum analysis was done for functional groups. The FT-IR spectra of drug with polymer were compared with the standard FT-IR spectrum of the pure drug.1658, 1611 (the vibrations characteristic to carboxyl group), $1578 \mathrm{~cm}-1(\mathrm{NH}$ bending group vibration) and $1546 \mathrm{~cm}-1$ for pure lisinopril compound are shifted to 1637 and $1592 \mathrm{~cm}-1$ for all IC products. These frequency shifts can be explained probably by breaking the hydrogen bonds in the case of $\mathrm{NH}$ groups 
Rathod Sayali P et al., Sch Acad J Pharm, Mar, 2021; 10(3): 54-59

and respectively by the formation of hydrogen bonds in the case of car-boxyl groups. Consequently, FTIR studies established clearly the functional groups implied in the inclusion process. In the 4000-2500 $\mathrm{cm}-1$ spectral region, see Fig. 4, the most important contributions are due to the $\mathrm{O}-\mathrm{H}$ stretching vibrations of primary (3504 $\left.\mathrm{cm}^{-1}[9]\right)$, secondary $\mathrm{OH}$ groups of the groups and soft water molecules present inside CD cavity, also. The different shape of this mass if (corresponding to ICs').

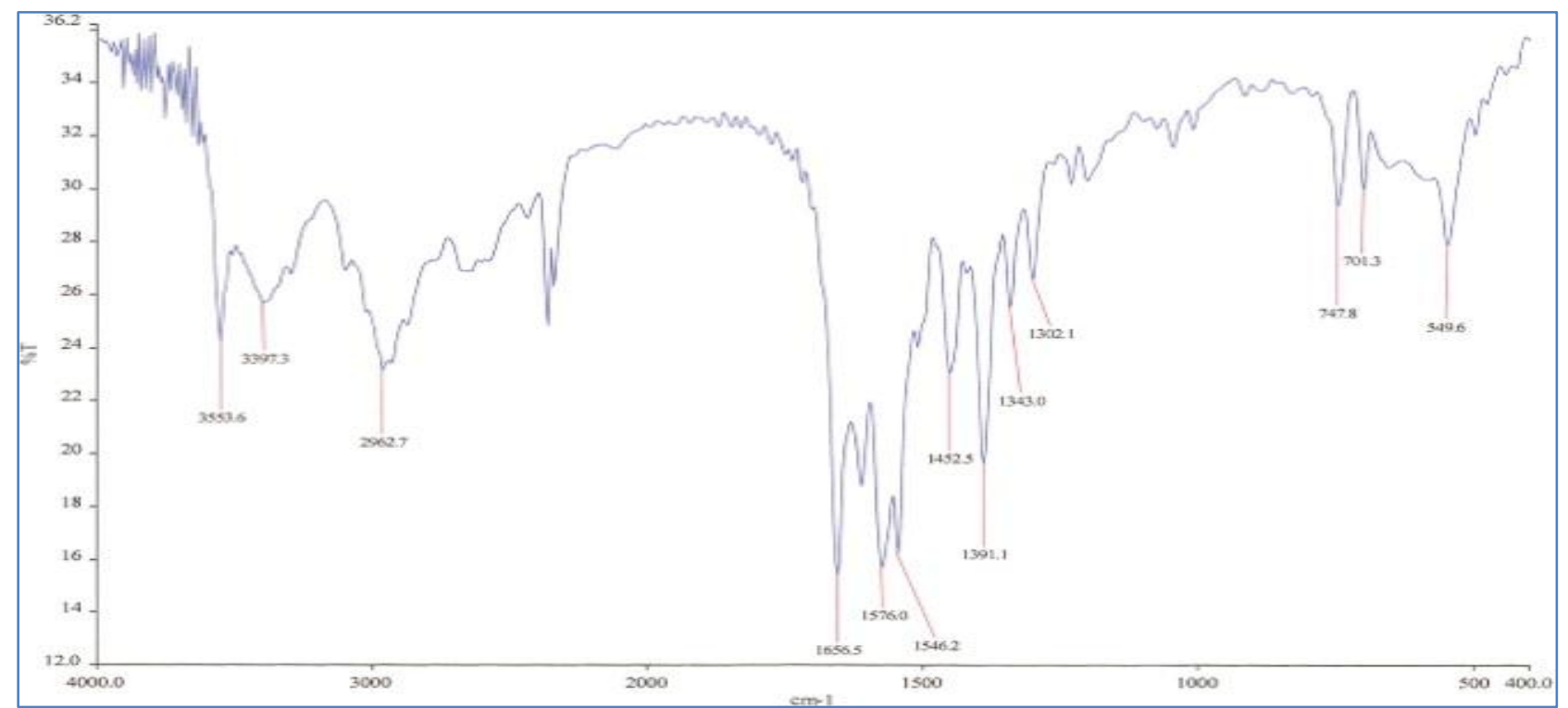

Fig-2: FTIR of Lisinopril

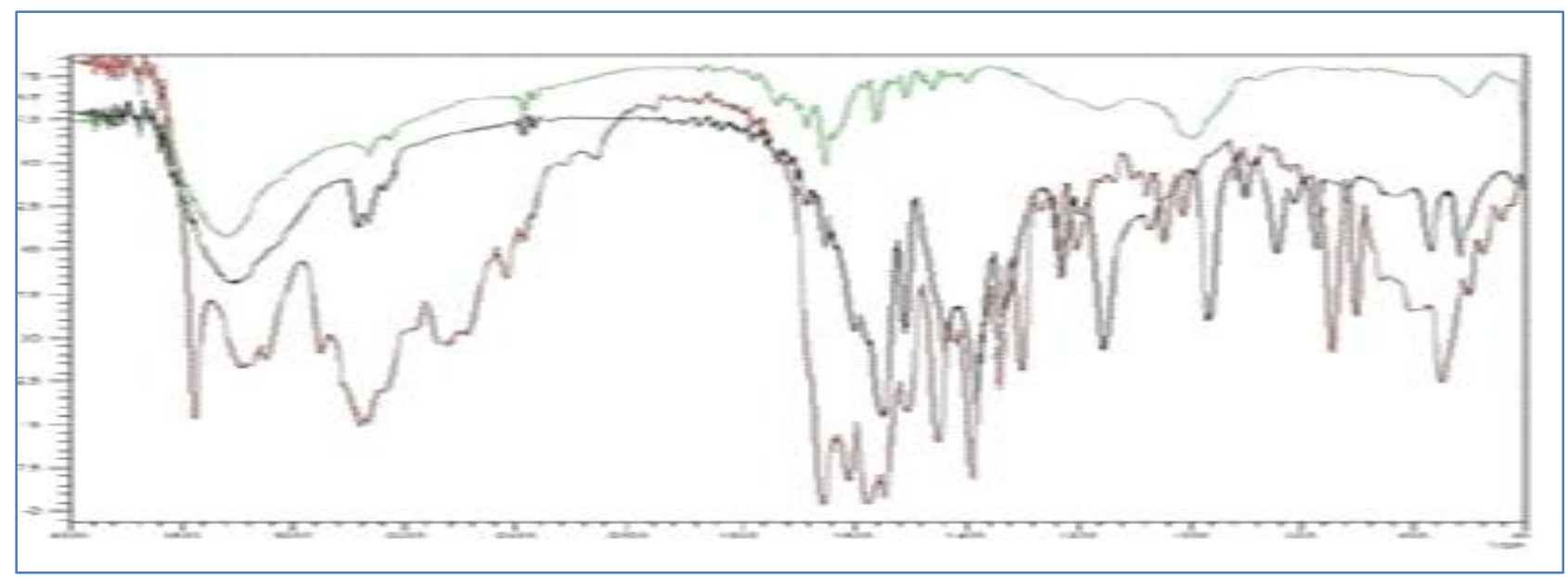

Fig-3: FTIR of Lisinopril physical mixture

\section{CONCLUSION}

Intragastric floating drug delivery system was prepared by incorporating low density materials, e.g. oils and/or waxes. Incorporation of wax into the beads influenced the drug release. It can be concluded that the drug Lisinopril multiparticulate floating beads for the treatment of hypertension were prepared and the release kinetics of the optimized batch could show the assured sustain release of drug. By using waxes it was concluded that the increase of drug release of Lisinopril could be obtained upto $10 \mathrm{hrs}$.Various preformulation studies like bulk density, tapped density, Carr's index angle of repose were in the acceptable limits especially for batch F9. Drug and polymer are compatible with each other as they are verified through FTIR spectroscopy. Increasing the amount of wax in the formulation significantly prolonged the drug release but was insufficient for sustaining the release of highly water-soluble drug.

\section{REFRENCES}

1. Arora S, Ali J, Khar RK, Baboota S, Floating drug delivery systems: A review, AAPS Pharm Sci Tech. 2005;6(3): 372-390.

2. Babu VBM, Khar RK, In vitro and In vivo studies of sustained release floating dosage forms containing salbutamol sulphate, Pharmazie. 1990; 45: 268-270.

3. Bardonnet PL, Faivre V, Pugh WJ, Piffaretti JC and Falson F, Gastro retentive Dosage Forms: 
Rathod Sayali P et al., Sch Acad J Pharm, Mar, 2021; 10(3): 54-59

Overview and Special case of Helicobacter pylori Journal of Control Release. 2006; 111.

4. Bari PH. A Comprehensive review on gastro retentive drug delivery system, IPP. 5(2):94102,2017

5. Chandiran S, Kumar BP and Narayan V., Formulation and in vitro evaluation of floating drug delivery system for salbutamol sulphate, International Journal of Pharma Biomed Sciences. 2010; 1(1): 12-15.

6. Chein YW, "Novel Drug Delivery System" 2nd ed. Marcel decker Inc., New York, 1-3.

7. Cook JD, Carriaga M, Kahn SG, Schalch W, Skikne BS, Gastric delivery system for iron supplementation, Lancet. 1990; 335: 1136-1139.

8. Abdul Sayeed, Gastro retentive Drug Delivery Systems: A Review, Der Pharmacia Lettre. 2011, 3(1): 121-137.

9. Streubel A, Siepmann J, Bodmeier R. "Floating microparticles based on low density foam powder", Int J Pharm. 2002; 241:279-92.

10. Desai S, Bolton S, A floating controlled release drug delivery system: in vitro- in vivo evaluation. Pharm Res. 1993, 10(9): 1321-1325.

11. Desai S. A Novel Floating Controlled Release Drug Delivery System Based on a Dried Gel Matrix Network [master's thesis]. Jamaica, NY: St John's University; 1984.

12. Dixit N, Floating Drug Delivery -System, Journal of Current Pharmaceutical Research. 2011; (1): 620.

13. Tekade BW. Optimization and in-vitro evaluation of verapamil hydrochloride floating tablet. The pharma innovation. 2014,3(6): 42-48

14. Garg S and Sharma S, Gastroretentive Drug Delivery System, Business Briefing: Pharma tech. 2003; 7:160-166.
15. Tekade BW, Jadhao UT, Thakare VM, Bhortake LR. Formulation and evaluation of diclofenac sodium effervescent tablet. Infrared Spectroscopy. 2014;9(10):11.

16. Geetha A, Kumar J Rajendra and Mohan $\mathrm{CH}$ Krishna, Review on: Floating drug delivery systems, International journal of pharmaceutical research and biomedical analysis. 2012; (1): 1-13.

17. Grubel P, Gastric emptying of non-digestible solids in the fasted dog, J Pharm Sci., $\quad 1987 ; 76: 117-$ 122.

18. Jadhao UT, Effect of Excipients and Process Variables over Gastro Retentive Antihypertensive Dosage Form, International Journal of Pharmaceutical Research \& Analysis. 2014; (4)3;186-192.

19. Jadhao UT. Design and Evaluation of Famotidine Matrix Tablet Using $3^{2}$ Factorial Designs, RJPBCS. 2013;3(4),1441-1451.

20. Jadhav CM, Bendale AN, Patil VR, Tekade B W, Thakare VM, Formulation and Evaluation of Effervescent Floating Tablet of Felodipine, IJPRD. 2012; 3(12): 43-48.

21. Jain A. New Concept: Floating Drug Delivery System, Indian Journal of Novel Drug Delivery. 2011; 3(3): 163-69.

22. Watson DG. Pharmaceutical Analysis A textbook for pharmacy students and pharmaceutical chemists, first ed. London, Churchill Livingstone. 1999: 100-03.

23. Duerst M. Spectroscopic methods of analysis: infrared spectroscopy. Swarbrick J., Boylon JC, Encyclopedia of Pharmaceutical Technology. 2007; 3:3405-18.

24. Skoog DA, Holler FJ, Nieman TA. Principles of Instrumental Analysis. 5 th ed. Sounder's College Publishing. 2004: 798- 808. 